\title{
DETERMINAÇÃO DE ENXOFRE EM SHAMPOO POR ESPECTROFOTOMETRIA UV-VIS: AVALIAÇÃO DE MÉTODOS DE PREPARO DE AMOSTRAS
}

Natalia J. Bielemann, Diogo L. R. Novo, Rodrigo M. Pereira, Julia E. Mello, Vanize C. Costa e Marcia F. Mesko*

Centro de Ciências Químicas, Farmacêuticas e de Alimentos, Universidade Federal de Pelotas, Campus Capão do Leão, 96160000, Capão do Leão - RS, Brasil

Recebido em 17/01/2017; aceito em 21/03/2017; publicado na web em 15/05/2017

\begin{abstract}
DETERMINATION OF SULFUR IN SHAMPOO BY UV-VIS SPECTROPHOTOMETRY: EVALUATION OF SAMPLE PREPARATION METHODS. Dry ashing, dissolution in aqueous medium or wet digestion methods were evaluated for sample preparation of shampoo for the subsequent indirect determination of sulfur by UV-Vis spectrophotometry. Among the sample preparation methods evaluated, microwave-assisted wet digestion using $7 \mathrm{~mol} \mathrm{~L}^{-1} \mathrm{HNO}_{3}$ was the most suitable method for this purpose. Accuracy of the proposed method was evaluated by recovery test using standard solution, and the recovery was about $96 \%$. Moreover, for comparison of results, $\mathrm{S}$ (as $\mathrm{SO}_{4}{ }^{2-}$ ) was also determined by ion chromatography, after microwave-assisted wet digestion using concentrated $\mathrm{HNO}_{3}$, and no significant difference was observed between the obtained results (according to the $t$-test for a $95 \%$ confidence level, $\mathrm{p}=0.288$ ). Relative standard deviations were always lower than $10 \%$ and the limit of detection was $0.007 \% \mathrm{w} / \mathrm{v}$. Finally, the proposed method was applied for analysis of sixteen shampoos used to many types of hair, from different brands produced in Brazil, and the concentration of $\mathrm{S}$ ranged from 0.78 to $2.82 \% \mathrm{w} / \mathrm{v}$.
\end{abstract}

Keywords: sulfur determination; shampoo; sample preparation; microwave-assisted wet digestion; UV-Vis spectrophotometry.

\section{INTRODUÇÃO}

Os shampoos têm sido amplamente utilizados como produto de beleza e de higiene pessoal visando à limpeza dos fios e do couro cabeludo. ${ }^{1,2}$ Atualmente, existe uma grande variedade desses produtos no mercado, os quais são destinados a diferentes tipos de cabelo, e apresentam propriedades que proporcionam aos usuários diferentes resultados após a sua utilização e, em alguns casos, ações farmacológicas. ${ }^{3}$ De acordo com os últimos dados declarados no ano de 2014 pela Associação Brasileira da Indústria de Higiene Pessoal, Perfumaria e Cosméticos (ABIHPEC), o mercado brasileiro de produtos para cabelos faturou em torno de R $\$ 21,2$ bilhões, sendo o shampoo, um dos produtos mais comercializados. ${ }^{4}$

Dentre os principais constituintes dos shampoos, destaca-se o enxofre (S), uma vez que é considerado um agente terapêutico quando aplicado em diversos cosméticos. Além disso, o S apresenta ações importantes para o cabelo como, por exemplo, queratolítica quando utilizado em elevadas concentrações, e queratoplástica quando aplicado em baixas concentrações. ${ }^{5}$ Ainda, cabe mencionar que os shampoos que apresentam $\mathrm{S}$ elementar e na forma de sulfacetamida podem ser eficazes no tratamento de rosácea, sarna, pitiríase versicolor, além de, geralmente, serem eficientes no tratamento de distúrbios capilares mais comuns, como a caspa, dermatite seborreica e oleosidade. ${ }^{6,7}$

O S também é adicionado aos shampoos como constituinte de surfactantes. Essas substâncias são amplamente utilizadas na produção de shampoos, pois auxiliam na remoção de resíduos, bem como na oleosidade presente no cabelo. ${ }^{8,9}$ Dentre os surfactantes mais utilizados, destacam-se o lauril sulfato de sódio e o lauril éter sulfato de sódio, ambos surfactantes aniônicos que apresentam alta detergência, facilidade de produção e menor custo. ${ }^{9,10}$

Apesar dos benefícios que o $\mathrm{S}$ proporciona para o cabelo, bem como para o couro cabeludo, a sua utilização é contraindicada para pessoas que apresentam hipersensibilidade a este elemento, e o contato com os olhos deve ser evitado. Ademais, uma elevada

*e-mail: marcia.mesko@pq.cnpq.br concentração de $\mathrm{S}$ em shampoos pode causar aos usuários dermatite alérgica de contato, irritações na pele e danos a estática dos fios, ocasionando o ressecamento do cabelo, independente da espécie química contendo $\mathrm{S}$ adicionada ao produto. ${ }^{11}$ Cabe ressaltar que a Food and Drug Administration (FDA) recomenda que a concentração de $\mathrm{S}$ adicionada a cosméticos que entrem em contato com o couro cabeludo não exceda a 5\%. Entretanto, quando o cosmético apresentar como principal finalidade o controle de caspas, é importante que a concentração de $\mathrm{S}$ esteja entre 2 e $5 \% .^{12}$

Assim, tendo em vista os benefícios e malefícios que podem advir da utilização de shampoos contendo uma concentração inadequada de $\mathrm{S}$, torna-se indispensável o controle da concentração deste elemento para garantir a qualidade do produto. Ainda, é importante salientar que não foram encontrados relatos de métodos na literatura para a determinação de $\mathrm{S}$ em shampoos.

Dentre as diversas técnicas analíticas que podem ser utilizadas para a determinação de $\mathrm{S}$, pode-se destacar a espectrometria de emissão óptica com plasma indutivamente acoplado (ICP-OES, do inglês Inductively Coupled Plasma Optical Emission Spectroscopy), ${ }^{13}$ a cromatografia de íons (IC, do inglês Ion Chromatography) ${ }^{13}$ e a espectrofotometria na região do ultravioleta e visível (UV-Vis, do inglês, Ultraviolet and Visible Spectrophotometry). ${ }^{14,15}$ No entanto, embora sejam técnicas bastante sensíveis para a determinação de S, a ICP-OES e a IC apresentam elevado custo de aquisição e manutenção quando comparadas com a espectrofotometria UV-Vis. Nesse sentido, tendo em vista o baixo custo operacional, robustez, rapidez de análise, confiabilidade nos resultados e fácil manuseio, a espectrofotometria UV-Vis pode ser uma interessante alternativa para a determinação de $\mathrm{S}$ em shampoo. ${ }^{14,15}$

A determinação de $\mathrm{S}$ por espectrofotometria UV-Vis é comumente realizada através de uma análise turbidimétrica. ${ }^{16}$ Contudo, para que isso seja possível, faz-se necessário que a amostra seja preparada de maneira adequada, pois a presença de compostos oriundos da matriz da amostra pode dificultar a formação do precipitado ou conferir turbidez a solução, culminando em resultados errôneos. Nesse contexto, é importante ressaltar que, apesar de ser uma amostra líquida, o shampoo apresenta grande quantidade de ingredientes 
com longas cadeias carbônicas, os quais podem interferir na etapa de determinação. Portanto, a realização de uma etapa de preparo de amostras, visando disponibilizar os analitos a partir da matriz da amostra para uma solução preferencialmente livre de interferências e adequada a técnica de determinação, é essencial para o êxito da análise. ${ }^{5,17}$

Embora a dissolução do shampoo em meio aquoso permita que os constituintes estejam presentes na solução, interferências relacionadas à matriz da amostra e a possibilidade do analito não estar disponível para ser detectado podem comprometer a exatidão dos resultados. Diante disso, dentre os métodos comumente utilizados para o preparo de amostras de shampoo, pode-se destacar a decomposição por via seca em forno mufla ${ }^{18} \mathrm{e}$ a decomposição por via úmida com aquecimento convencional ${ }^{19}$ ou assistida por radiação micro-ondas. ${ }^{20,21}$ No entanto, é importante mencionar que esses métodos de preparo de amostras ainda não foram utilizados para a posterior determinação de $\mathrm{S}$ em shampoo.

A decomposição por via seca em forno mufla apresenta diversas vantagens, principalmente, devido a decomposição completa da matéria orgânica, bem como pela possibilidade de reduzir os limites de detecção do método, uma vez que é realizada em sistema aberto, permitindo a decomposição de elevadas massas de amostra. No entanto, o procedimento necessita demasiado tempo de execução, além de ser bastante suscetível a contaminações e perdas de elementos por volatilização e/ou adsorção nas paredes do recipiente utilizado durante o processo. ${ }^{22,23}$ Por outro lado, a decomposição por via úmida promove a oxidação da matéria orgânica da amostra através do uso de um ácido oxidante, de uma mistura de ácidos oxidantes ou de uma mistura contendo um ácido oxidante e peróxido de hidrogênio. Contudo, quando realizada em sistemas abertos a temperatura de decomposição é limitada ao ponto de ebulição dos reagentes. Nesse sentido, geralmente é requerido um elevado tempo de decomposição, bem como uma elevada quantidade de reagentes para a total oxidação da matéria orgânica, principalmente daqueles que apresentam temperaturas de ebulição mais elevadas como, por exemplo, o $\mathrm{H}_{2} \mathrm{SO}_{4} \cdot{ }^{22-24} \mathrm{Assim}$, a utilização de sistemas de decomposição com frascos fechados associado ao aquecimento com radiação micro-ondas tem sido uma tendência para o preparo de amostras que apresentam composição predominantemente orgânica, bem como para amostras de shampoo. ${ }^{6}$ Apesar de permitir o uso de massas de amostras menores quando comparado aos métodos com fracos abertos, a utilização de frascos fechados possibilita o uso de menores quantidades de reagentes sem comprometer a eficiência de decomposição. Isso está associado a redução na perda dos reagentes por volatilização, por se tratar de um sistema fechado, bem como pela possibilidade de realizar a decomposição das amostras sob elevadas pressões, o que resulta em um aumento no ponto de ebulição dos reagentes e, consequentemente, da eficiência de oxidação da matéria orgânica. Além disso, a possibilidade de trabalhar com sistemas de decomposição com frascos fechados minimiza a ocorrência de perdas e contaminações durante o preparo das amostras. ${ }^{25,26}$

Diante disso, esse trabalho teve como objetivo avaliar a decomposição por via seca em forno mufla, bem como a dissolução em meio aquoso e a decomposição por via úmida, ambas em sistemas com frascos abertos ou fechados, com aquecimento convencional ou assistido por radiação micro-ondas, visando o preparo de amostras de shampoo para a subsequente determinação indireta de S por espectrofotometria UV-Vis. A exatidão do método considerado mais adequado foi avaliada através de ensaios de recuperação, bem como pela comparação dos resultados com os obtidos após a determinação por IC. Por fim, foi realizada a determinação de $\mathrm{S}$ em shampoos de variados tipos e marcas utilizando o método proposto.

\section{PARTE EXPERIMENTAL}

\section{Amostras}

Os shampoos utilizados nesse trabalho foram adquiridos no comércio local da cidade de Pelotas/RS e são destinados a variados tipos de cabelos (lisos, ondulados, cacheados e tingidos) e finalidades (controle de oleosidade, para cabelos secos, redução de frizz ou caspas). As amostras utilizadas foram provenientes de diferentes marcas da indústria brasileira. Além disso, vale mencionar que dentre as dezesseis amostras utilizadas, duas são comercializadas como produtos isentos de $\mathrm{S}$ na forma de $\mathrm{SO}_{4}{ }^{2-}$. As otimizações iniciais foram realizadas utilizando um shampoo destinado a cabelos normais e oleosos, o qual foi selecionado aleatoriamente, e as outras amostras foram, posteriormente, analisadas utilizando o método proposto.

\section{Instrumentação}

Para a realização da decomposição do shampoo por via seca, um forno tipo mufla (2000G, Tekpluz, Brasil) foi utilizado, enquanto, a solubilização das cinzas foi realizada com o auxílio de um agitador magnético (C-MAG HS 7, IKA ${ }^{\circledR}$, Alemanha). Os procedimentos de dissolução em meio aquoso e decomposição por via úmida em frasco aberto com aquecimento convencional foram realizados em um bloco digestor (MA4025, Marconi, Brasil) equipado com oito frascos de vidro de borossilicato $(50 \times 250 \mathrm{~mm})$.

Por sua vez, a dissolução em meio aquoso e a decomposição por via úmida assistida por radiação micro-ondas em frascos fechados foram realizadas em um forno de micro-ondas (Multiwave $3000^{\circledR}$, Anton Paar, Áustria), equipado com oito frascos de politetrafluoretileno modificado quimicamente (PTFE-TFM ${ }^{\circledR}$ ) com volume máximo de $100 \mathrm{~mL}$ e, temperatura e pressão de trabalho em torno de $260{ }^{\circ} \mathrm{C}$ e 60 bar, respectivamente.

Enxofre foi determinado como $\mathrm{SO}_{4}{ }^{2-}$ utilizando um espectrofotômetro (IL-592, Kasuaki, Brasil) que opera na faixa UV-Vis de 190 a 1100 nm e apresenta banda de passagem de $2 \mathrm{~nm}$. Ainda, para comparação dos resultados, $\mathrm{S}$ também foi determinado como $\mathrm{SO}_{4}{ }^{2-}$ utilizando um cromatógrafo de íons (861 IC Advanced Compact, Metrohm, Suiça) equipado com sistema de supressão química - Metrohm Suppressor Module (MSM) - e detecção por condutividade. Para tanto, foi utilizada uma coluna de troca aniônica com grupos amônio quaternário suportados em poli(álcool vinílico), modelo Metrosep A Supp 5 (250 x 4 mm d.i., 5 m de diâmetro de partícula, Metrohm), e uma coluna-guarda de mesmo material de preenchimento da coluna de troca iônica, modelo Metrosep A Supp 4/5 Guard (5 x 4 mm d.i., 5 um de diâmetro de partícula, Metrohm). A alça de amostragem e a vazão do eluente utilizados neste trabalho foram de $25 \mu \mathrm{L}$ e $0,7 \mathrm{~mL} \mathrm{~min}^{-1}$, respectivamente.

\section{Reagentes e soluções}

Todos os reagentes utilizados neste trabalho foram de grau analítico (Merck, Alemanha) e as soluções foram preparadas com água ultrapura $(18,3 \mathrm{M} \Omega \mathrm{cm})$ obtida através de um sistema de ultrapurificação (MEGA UP, MegaPurity, Coreia do Sul). Água ultrapura também foi utilizada para a lavagem de materiais e vidrarias após a descontaminação utilizando ácido nítrico $10 \%$ (v/v) ou concentrado. Por sua vez, o $\mathrm{HNO}_{3} 65 \%$ (m/m) utilizado nos métodos de decomposição por via úmida foi previamente purificado em um sistema de destilação abaixo do ponto de ebulição - em torno de $65^{\circ} \mathrm{C}$ (Milestone, Model Duopor, Itália).

Para a calibração dos equipamentos e para a realização dos ensaios de recuperação, uma solução estoque de $\mathrm{SO}_{4}{ }^{2-}\left(10000 \mathrm{mg} \mathrm{L}^{-1}\right)$ foi preparada a partir da dissolução do sal de sulfato de sódio em água. 
Para a análise por espectrofotometria UV-Vis uma curva de calibração de 5 a $30 \mathrm{mg} \mathrm{L}^{-1}$ de $\mathrm{SO}_{4}{ }^{2-}$ foi preparada a partir da diluição da solução estoque de $\mathrm{SO}_{4}{ }^{2-}\left(1000 \mathrm{mg} \mathrm{L}^{-1}\right)$ e da adição de cloreto de bário $10 \%$ $(\mathrm{m} / \mathrm{v})$ e de ácido clorídrico $0,5 \mathrm{~mol} \mathrm{~L}^{-1}$, os quais foram utilizados para promover a formação de precipitado. Por outro lado, para a análise

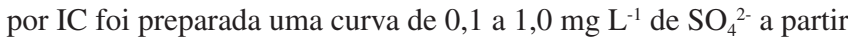
da diluição da solução estoque de $\mathrm{SO}_{4}{ }^{2-}$. O eluente utilizado para a determinação de $\mathrm{SO}_{4}{ }^{2-}$ por IC $\left[\mathrm{Na}_{2} \mathrm{CO}_{3}\left(3,2 \mathrm{mmol} \mathrm{L}{ }^{-1}\right) / \mathrm{NaHCO}_{3}(1,0\right.$ mmol $\left.\mathrm{L}^{-1}\right)$ ] foi preparado pela dissolução dos seus respectivos sais em água. Uma solução de ácido sulfúrico na concentração de 200 mmol L-1 e água foram utilizadas como soluções regeneradoras do sistema de supressão química. Para avaliar a acidez dos digeridos, foi realizada uma titulação ácido/base utilizando hidróxido de sódio $0,09474 \mathrm{~mol} \mathrm{~L}^{-1}$ previamente padronizado com biftalato de potássio

\section{Decomposição de shampoo assistida por radiação micro-ondas e determinação indireta de enxofre por cromatografia de íons}

A decomposição por via úmida assistida por radiação micro-ondas em frasco fechado, utilizando $\mathrm{HNO}_{3} 14 \mathrm{~mol} \mathrm{~L}^{-1}$ como solução digestora, foi utilizada como método de referência previamente a determinação por IC. Para isso, $0,5 \mathrm{~mL}$ de shampoo, o qual foi escolhido aleatoriamente, foi transferido para os frascos de reação, aos quais foram adicionados $6 \mathrm{~mL}$ de $\mathrm{HNO}_{3} 14 \mathrm{~mol} \mathrm{~L}^{-1}$. Posteriormente, os frascos foram fechados, fixados ao rotor e submetidos ao seguinte programa de irradiação com micro-ondas: $i$ ) rampa de 10 min até $1000 \mathrm{~W}$; ii) $1000 \mathrm{~W}$ por $10 \mathrm{~min}$; iii) $0 \mathrm{~W}$ por $20 \mathrm{~min}$ (etapa de resfriamento). Finalizadas as etapas de aquecimento e resfriamento, as soluções obtidas foram transferidas para balões volumétricos de $25 \mathrm{~mL}$ e o volume foi aferido com água. Por fim, $\mathrm{S}$ foi determinado na forma de $\mathrm{SO}_{4}{ }^{2-}$ por $\mathrm{IC}$, e os resultados obtidos por este método foram utilizados para fins de comparação com os obtidos utilizando os demais métodos avaliados.

A exatidão do método usado como referência e do método proposto foi avaliada através de ensaios de recuperação, os quais foram realizados a partir da adição de $900 \mu \mathrm{L}$ de uma solução estoque contendo $10000 \mathrm{mg} \mathrm{L}^{-1}$ de $\mathrm{SO}_{4}{ }^{2-}$ a amostra, previamente a etapa de decomposição. Além disso, os digeridos obtidos pelo método proposto também foram analisados por IC para a comparação dos resultados.

\section{Decomposição de shampoo por via seca utilizando um forno tipo mufla}

Inicialmente, cerca de $2 \mathrm{~mL}$ de shampoo foram transferidos para cadinhos de porcelana, os quais foram submetidos ao seguinte programa de aquecimento em um forno tipo mufla: i) $30 \mathrm{~min}$ a $90^{\circ} \mathrm{C}$; ii) $60 \mathrm{~min}$ a $500{ }^{\circ} \mathrm{C}$. Posteriormente, as cinzas foram solubilizadas com $500 \mu \mathrm{L}$ de $\mathrm{HNO}_{3} 14 \mathrm{~mol} \mathrm{~L}^{-1}$ e $5 \mathrm{~mL}$ de água. Por fim, as soluções foram filtradas e transferidas para um balão volumétrico de $25 \mathrm{~mL}$ e avolumadas com água ultrapura. Essas condições foram selecionadas com base no trabalho desenvolvido por Salvador et al., ${ }^{6}$ que descreve um método para determinação de selênio, zinco e cádmio em shampoo antiscaspas, por espectrometria atômica.

\section{Preparo de amostra de shampoo utilizando aquecimento convencional em frasco aberto}

Inicialmente, para a realização da dissolução em meio aquoso utilizando aquecimento convencional em sistema aberto, cerca de $0,5 \mathrm{~mL}$ de shampoo foi transferido para frascos do bloco digestor sendo, posteriormente, adicionados $20 \mathrm{~mL}$ de água ultrapura. Para a decomposição por via úmida, $0,5 \mathrm{~mL}$ de shampoo foi transferido para os frascos, no entanto, $20 \mathrm{~mL}$ de $\mathrm{HNO}_{3}$ em diferentes concentrações (4, 7 ou $\left.14 \mathrm{~mol} \mathrm{~L}^{-1}\right)$ foram adicionados nos frascos de decomposição.
Em ambos os procedimentos, as amostras foram submetidas a aquecimento a $80^{\circ} \mathrm{C}$ por $2 \mathrm{~h}$ e as soluções obtidas foram transferidas para balões volumétricos de $25 \mathrm{~mL}$ e aferidas com água.

\section{Preparo de amostra de shampoo utilizando aquecimento com} radiação micro-ondas em frasco fechado

A dissolução em meio aquoso e a decomposição por via úmida utilizando $\mathrm{HNO}_{3}\left(1,4,7\right.$ ou $\left.14 \mathrm{~mol} \mathrm{~L}^{-1}\right)$ foram realizadas em sistema com frascos fechados assistido por radiação micro-ondas. Em um primeiro momento, cerca de $0,5 \mathrm{~mL}$ de shampoo foi transferido para frascos de reação, aos quais foram adicionados $6 \mathrm{~mL}$ de água ultrapura ou de $\mathrm{HNO}_{3}$ nas concentrações avaliadas. Posteriormente, os frascos foram fechados, fixados ao rotor e submetidos ao programa de irradiação com micro-ondas conforme descrito no item "Decomposição de shampoo assistida por radiação micro-ondas e determinação indireta de enxofre por cromatografia de íons". Após finalizado o programa, as soluções obtidas foram transferidas para balões volumétricos de $25 \mathrm{~mL}$ e o volume foi aferido com água.

\section{Determinação indireta de S em shampoo por espectrofotometria UV-Vis}

Para a determinação de S por espectrofotometria UV-Vis através de análise turbidimétrica, as soluções provenientes dos diversos métodos de preparo de amostras foram diluídas em um balão volumétrico de $25 \mathrm{~mL}$, sendo adicionados $500 \mu \mathrm{L}$ da amostra obtida após dissolução ou decomposição, $2 \mathrm{~mL}$ de $\mathrm{HCl} 0,5$ mol L-1 e $1 \mathrm{~mL}$ de $\mathrm{BaCl}_{2}$ $10 \%(\mathrm{~m} / \mathrm{v})$. Posteriormente, as soluções foram homogeneizadas e a determinação de $\mathrm{S}$ como $\mathrm{SO}_{4}{ }^{2-}$ foi realizada no comprimento de onda de $420 \mathrm{~nm}$. Essas condições foram utilizadas com base no trabalho desenvolvido por Tabatai. ${ }^{16}$

\section{RESULTADOS E DISCUSSÃO}

Determinação indireta de $\mathrm{S}$ em shampoo por cromatografia de íons após decomposição assistida por radiação micro-ondas

Com o intuito de obter um valor que pudesse ser utilizado para comparação dos resultados, tendo em vista que não existem métodos oficiais ou relatos na literatura para a determinação de $\mathrm{S}$ em shampoo, a amostra S1 foi decomposta em um sistema com frasco fechado assistido por radiação micro-ondas com $6 \mathrm{~mL}$ de $\mathrm{HNO}_{3} 14 \mathrm{~mol} \mathrm{~L}{ }^{-1}$, conforme descrito na seção "Parte experimental" e, posteriormente, os digeridos foram analisados por IC para a determinação de $\mathrm{S}$ na forma de $\mathrm{SO}_{4}{ }^{2-}$. Esse método foi utilizado como referência, visto que proporciona condições adequadas para a eficiente decomposição da matéria orgânica, além de apresentar menores riscos de perda por volatilização e contaminação quando comparado aos métodos convencionais de digestão. ${ }^{26} \mathrm{~A}$ determinação de $\mathrm{S}$ foi realizada por $\mathrm{IC}$, pois essa técnica apresenta sensibilidade adequada, além de ser consolidada para esse tipo de análise e amplamente utilizada para determinação de $\mathrm{S}$ na forma de $\mathrm{SO}_{4}{ }^{2-}$ em diferentes tipos de amostra. ${ }^{27-29}$ Todavia, cabe mencionar que a determinação de $\mathrm{S}$ por IC nesses digeridos somente foi possível devido à elevada concentração deste analito na amostra avaliada. Isso permitiu a realização de diluições de até 2000 vezes, minimizando os efeitos da elevada acidez residual dos digeridos e também da elevada concentração de íons nitrato, os quais poderiam inviabilizar a determinação de S por IC.${ }^{13}$ A exatidão do método foi avaliada através da realização de ensaios de recuperação utilizando solução padrão, e recuperações obtidas foram em torno de $98 \pm 9 \%$. Assim, a concentração de S obtida para a amostra S1 (1,04 $\pm 0,10 \%$ $\mathrm{m} / \mathrm{v}$ ) foi adotada como referência para a avaliação dos demais métodos. 


\section{Avaliação de métodos de preparo de amostra visando a posterior determinação de $\mathrm{S}$ em shampoo}

Após a obtenção de um valor de referência para a concentração de $\mathrm{S}$ na amostra de shampoo, os métodos de preparo de amostras descritos na seção "Parte experimental" foram avaliados. Os resultados obtidos para S por espectrofotometria UV-Vis e o aspecto dos digeridos após a avaliação dos métodos de preparo de amostras estão descritos na Tabela 1 .

Como pode ser observado na Tabela 1, a maioria das soluções obtidas após o uso de diferentes métodos de preparo de amostra não foram compatíveis para a determinação de $\mathrm{S}$ em shampoo por espectrofotometria UV-Vis, tendo em vista que estas soluções apresentaram coloração, turbidez e/ou acidez nos digeridos inadequados a análise. Com relação a presença de coloração e turbidez na amostra, é importante salientar que estas podem influenciar na medida turbidimétrica, culminando em resultados inexatos, tendo em vista que somente a turbidez associada a formação do $\mathrm{BaSO}_{4}$ deve ser determinada. Por outro lado, a elevada acidez dos digeridos após a etapa de preparo da amostra pode aumentar a dissociação do precipitado de $\mathrm{BaSO}_{4}, \mathrm{O}$ que também pode influenciar na exatidão dos resultados..$^{30}$

Ainda, com base nos resultados obtidos, pode-se observar que quando realizado o preparo da amostra de shampoo através da decomposição por via seca utilizando forno tipo mufla, a solução resultante apresentou um aspecto límpido e adequado para a análise turbidimétrica (Figura 1A). No entanto, quando realizada a determinação de S por espectrofotometria UV-Vis a concentração obtida $(0,62 \pm$ $0,08 \% \mathrm{~m} / \mathrm{v}$ ) foi bastante inferior quando comparada com o valor de referência $(1,04 \pm 0,10 \% \mathrm{~m} / \mathrm{v})$. Provavelmente, a diferença observada pode estar associada a erros causados pela decomposição incompleta da amostra, uma vez que foi observada a presença de fuligem no cadinho após a solubilização das cinzas. Além disso, dependendo da composição da amostra, espécies voláteis de $\mathrm{S}$, como, por exemplo, $\mathrm{SO}_{2}$ e $\mathrm{SO}_{3}$, podem ser formadas durante o aquecimento e combustão da amostra, sendo perdidas por volatilização devido à utilização de recipientes abertos e elevadas temperaturas. ${ }^{13}$

Quando realizada a dissolução do shampoo em meio aquoso utilizando frasco aberto com aquecimento convencional, as soluções apresentaram formação de espuma e turbidez, o que pode influenciar na determinação turbidimétrica de $\mathrm{S}$ por espectrofotometria UV-Vis, como mencionado anteriormente (Figura 1B). Além disso, as soluções obtidas após a decomposição por via úmida com aquecimento convencional em frasco aberto utilizando $\mathrm{HNO}_{3}$ nas concentrações de 4 ou $7 \mathrm{~mol} \mathrm{~L}^{-1}$, também apresentaram aspecto inadequado devido a presença de turbidez e espuma, o que pode estar relacionado com a decomposição incompleta da matriz da amostra após a etapa de decomposição, não sendo possível realizar a determinação de $\mathrm{S}$ por análise turbidimétrica (Figura 1C e 1D). Por outro lado, quando utilizado $\mathrm{HNO}_{3}$ na concentração de $14 \mathrm{~mol} \mathrm{~L}^{-1}$, os digeridos apresentaram um aspecto límpido, conforme pode ser verificado na Figura 1E. Entretanto, após a adição do reagente precipitante $\left(\mathrm{BaCl}_{2}\right)$ não foi observada a formação do precipitado, pois a presença de elevada concentração de íons $\mathrm{H}^{+}$em solução pode favorecer a dissociação do $\mathrm{BaSO}_{4}$, aumentando a sua solubilidade em meio aquoso. ${ }^{30} \mathrm{Nessa}$ condição, a concentração de íons $\mathrm{H}^{+}$remanescentes em solução após o preparo da amostra utilizando a digestão por via úmida em sistema aberto com $\mathrm{HNO}_{3} 14 \mathrm{~mol} \mathrm{~L}^{-1}$ foi bastante elevada (cerca de 11,2 mol $\mathrm{L}^{-1}$ ), o que provavelmente favoreceu esse processo.

Com relação aos resultados obtidos utilizando a dissolução do shampoo em meio aquoso em frasco fechado assistido por radiação micro-ondas, as soluções também apresentaram coloração e espuma devido à dissolução de compostos presentes na matriz

Tabela 1. Concentração de $\mathrm{S}$ em shampoo e descrição do aspecto dos digeridos após a avaliação de diferentes métodos de preparo de amostra. Determinação por espectrofotometria UV-Vis ( $\% \mathrm{~m} / \mathrm{v}$, média \pm desvio padrão, $\mathrm{n}=3$ )

\begin{tabular}{|c|c|c|c|}
\hline Método de preparo da amostra & Solução dissolutora/digestora & Concentração de S & Aspecto da solução \\
\hline Via seca & - & $0,62 \pm 0,08$ & Límpido \\
\hline \multirow[t]{4}{*}{ Via úmida com frasco aberto } & $\mathrm{H}_{2} \mathrm{O}$ & $\mathrm{nd}^{*}$ & Túrbido e formação de espuma \\
\hline & $\mathrm{HNO}_{3} 4 \mathrm{~mol} \mathrm{~L}^{-1}$ & $\mathrm{nd}^{*}$ & Túrbido e formação de espuma \\
\hline & $\mathrm{HNO}_{3} 7 \mathrm{~mol} \mathrm{~L}^{-1}$ & $\mathrm{nd}^{*}$ & Túrbido e formação de espuma \\
\hline & $\mathrm{HNO}_{3} 14 \mathrm{~mol} \mathrm{~L}^{-1}$ & $\mathrm{nd}^{*}$ & Límpido \\
\hline \multirow[t]{5}{*}{ Via úmida com frasco fechado } & $\mathrm{H}_{2} \mathrm{O}$ & $\mathrm{nd}^{*}$ & Coloração escura e formação de espuma \\
\hline & $\mathrm{HNO}_{3} 1 \mathrm{~mol} \mathrm{~L}^{-1}$ & $\mathrm{nd}^{*}$ & Coloração verde \\
\hline & $\mathrm{HNO}_{3} 4 \mathrm{~mol} \mathrm{~L}^{-1}$ & nd* & Coloração verde \\
\hline & $\mathrm{HNO}_{3} 7 \mathrm{~mol} \mathrm{~L}^{-1}$ & $1,14 \pm 0,10$ & Límpido \\
\hline & $\mathrm{HNO}_{3} 14 \mathrm{~mol} \mathrm{~L}^{-1}$ & $0,39 \pm 0,05$ & Límpido \\
\hline
\end{tabular}

*Não determinado.

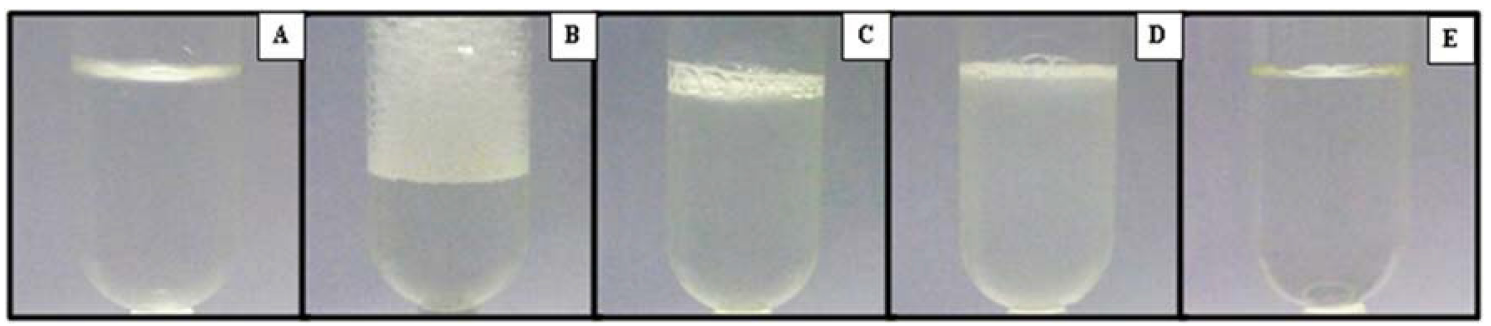

Figura 1. Aspectos das soluções obtidas após (A) decomposição do shampoo por via seca com a dissolução das cinzas utilizando 500 $\mu$ L HNO 14 mol L-1 e 5 $m L$ de água, (B) dissolução em meio aquoso e decomposição por via úmida em frasco aberto com aquecimento convencional utilizando (C) $\mathrm{HNO}_{3} 4$ mol $\mathrm{L}^{-1}$, (D) $\mathrm{HNO}_{3} 7 \mathrm{~mol} \mathrm{~L}^{-1} \mathrm{e}(\mathrm{E}) \mathrm{HNO}_{3} 14 \mathrm{~mol} \mathrm{~L}^{-1}$ 


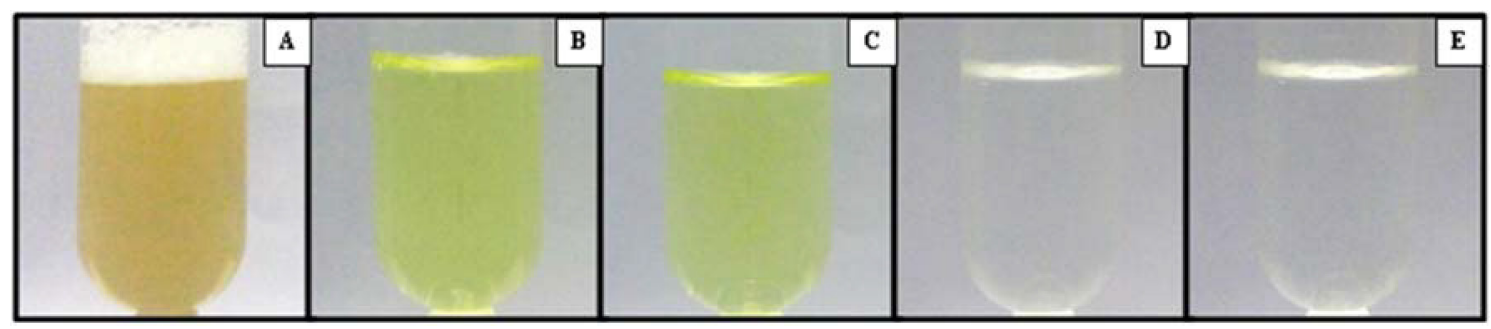

Figura 2. Aspectos das soluções obtidas após a (A) dissolução em meio aquoso e decomposição por via úmida em frasco fechado assistida por radiação micro-ondas utilizando (B) $\mathrm{HNO}_{3} 1 \mathrm{~mol} \mathrm{~L}^{-1}$, (C) $\mathrm{HNO}_{3} 4 \mathrm{~mol} \mathrm{~L}^{-1}$, (D) $\mathrm{HNO}_{3} 7 \mathrm{~mol} \mathrm{~L}^{-1}$ e (E) $\mathrm{HNO}_{3} 14 \mathrm{~mol} \mathrm{~L}^{-1}$

da amostra (Figura 2A). Além disso, as soluções obtidas após a decomposição por via úmida em frasco fechado assistido por radiação micro-ondas utilizando $\mathrm{HNO}_{3}$ nas concentrações de 1 e 4 mol L ${ }^{-1}$, também apresentaram aspecto inadequado para a análise turbidimétrica devido à presença de coloração esverdeada, o que pode estar relacionado a decomposição incompleta da matriz da amostra após a etapa de decomposição (Figura 2 B e C). Por outro lado, quando utilizado $\mathrm{HNO}_{3}$ na concentração de 7 e 14 mol L-1, os digeridos apresentaram um aspecto límpido, conforme pode ser verificado nas Figuras 2 D e E.

Embora a solução obtida após a digestão em sistema fechado utilizando $\mathrm{HNO}_{3}$ na concentração de $14 \mathrm{~mol} \mathrm{~L}^{-1}$ tenha apresentado um aspecto límpido (Figura 2E), o resultado obtido após a determinação de S por espectrofotometria UV-Vis $(0,39 \pm 0,05 \% \mathrm{~m} / \mathrm{v})$ apresentou diferença significativa quando comparado com o valor adotado como referência $(1,04 \pm 0,10 \% \mathrm{~m} / \mathrm{v})$. Isso possivelmente está associado a solubilização parcial do precipitado devido a concentração de íons $\mathrm{H}^{+}$remanescentes em solução após o preparo da amostra, que foi de $1,98 \mathrm{~mol} \mathrm{~L}^{-1}$. Visando contornar esse efeito, sucessivas diluições foram realizadas previamente a etapa de determinação. Contudo, não houve a formação de precipitado, provavelmente, em virtude da baixa concentração do analito em solução após realizar sucessivas diluições.

Por outro lado, é importante mencionar que os digeridos obtidos após a decomposição por via úmida assistida por radiação micro-ondas em frasco fechado utilizando $\mathrm{HNO}_{3} 7 \mathrm{~mol} \mathrm{~L}^{-1}$, além de terem apresentado aspecto límpido (Figura 2D), viabilizaram a obtenção de resultados por espectrofotometria UV-Vis $(1,14 \pm 0,10 \% \mathrm{~m} / \mathrm{v})$ concordantes com o valor adotado como referência $(1,04 \pm 0,10 \%$ $\mathrm{m} / \mathrm{v}$ ). Vale ressaltar que a concentração de íons $\mathrm{H}^{+}$remanescente em solução após o preparo da amostra nessa condição foi inferior (1,06 mol $\left.\mathrm{L}^{-1}\right)$ às observadas nas condições em que não houve a formação de precipitado (decomposição por via úmida com aquecimento convencional em sistema aberto utilizando $14 \mathrm{~mol} \mathrm{~L}^{-1}$ ), bem como na condição em que o resultado não foi concordante com o valor adotado como referência (decomposição por via úmida assistida por radiação micro-ondas em frasco fechado utilizando $14 \mathrm{~mol} \mathrm{~L}^{-1}$ ). Assim, diante desses resultados, a decomposição por via úmida assistida por radiação micro-ondas em frasco fechado utilizando $\mathrm{HNO}_{3}$ na concentração de $7 \mathrm{~mol} \mathrm{~L}^{-1}$ foi selecionada para os estudos subsequentes. Nesse contexto, é importante enfatizar que o método selecionado proporciona a obtenção de digeridos compatíveis com a técnica de determinação, além de envolver a utilização de frascos fechados, o que minimiza a ocorrência de contaminações e de perdas por volatilização.

\section{Avaliação da exatidão do método proposto}

Para avaliação da exatidão das condições selecionadas foram realizados ensaios de recuperação, nos quais foram obtidas recuperações em torno de $96 \pm 7 \%$. Desta forma, os resultados demonstram que, assim como o método de referência, a decomposição por via úmida assistida por radiação micro-ondas em sistema fechado utilizando ácido $7 \mathrm{~mol} \mathrm{~L}^{-1}$ e subsequente determinação de $\mathrm{S}$ em shampoo por espectrofotometria UV-Vis apresenta exatidão adequada.

Adicionalmente, os digeridos obtidos utilizando o método de preparo de amostra proposto foram analisados por cromatografia de íons, e a concentração de $S$ determinada não apresentou diferença significativa (de acordo com o test- $t$ Student ao nível de confiança de $95 \%, \mathrm{p}=0.288$ ) quando comparado com o valor obtido por espectrofotometria UV-Vis. Além disso, a concentração de $\mathrm{S}$ obtida $(1,14$ $\pm 0,10 \% \mathrm{~m} / \mathrm{v}$ ) foi concordante em $110 \%$ quando comparada com o valor de referência $(1,04 \pm 0,10 \% \mathrm{~m} / \mathrm{v})$.

Cabe mencionar que o desvio padrão relativo (RSD, do inglês Relative Standard Deviation) do método proposto foi inferior a $10 \%$, enquanto que o limite de detecção (LOD do inglês Limit of Detection) foi de $0,007 \% \mathrm{~m} / \mathrm{v}$, estando este de acordo para atender aos limites recomendados para S pela FDA. O LOD do método foi calculado de acordo com as recomendações do Instituto Nacional de Metrologia, Qualidade e Tecnologia (INMETRO). ${ }^{31}$

\section{Determinação de S em shampoo}

Como descrito anteriormente, o método de decomposição por via úmida assistida por radiação micro-ondas em frasco fechado utilizando $\mathrm{HNO}_{3}$ na concentração de $7 \mathrm{~mol} \mathrm{~L}^{-1}$ foi selecionado para a decomposição de variados tipos de shampoos visando à determinação de S por espectrofotometria UV-Vis. Os resultados obtidos são apresentados na Tabela 2.

Tabela 2. Concentrações de $\mathrm{S}$ em shampoos após decomposição por via úmida assistida por radiação micro-ondas em frasco fechado utilizando $\mathrm{HNO}_{3}$ $7 \mathrm{~mol} \mathrm{~L}^{-1}$ e determinação por espectrofotometria UV-Vis $(\% \mathrm{~m} / \mathrm{v}$, média \pm desvio padrão, $\mathrm{n}=3$ )

\begin{tabular}{lc}
\hline Shampoo (finalidade) & $\mathrm{S}$ \\
\hline S1 (cabelos normais e controle de oleosidade) & $1,14 \pm 0,10$ \\
S2 (realce de cor) & $0,84 \pm 0,08$ \\
S3 (antirresíduos) & $0,81 \pm 0,07$ \\
S4 (realce de cor) & $1,51 \pm 0,14$ \\
S5 (controle de oleosidade) & $0,78 \pm 0,07$ \\
S6 (realce de cor) & $1,44 \pm 0,09$ \\
S7 (controle de queda) & $2,06 \pm 0,15$ \\
S8 (reconstrução de fios) & $2,08 \pm 0,04$ \\
S9 (controle de oleosidade) & $2,49 \pm 0,09$ \\
S10 (cabelos normais) & $1,31 \pm 0,06$ \\
S11 (reconstrução de fios) & $1,80 \pm 0,11$ \\
S12 (cabelos ressecados) & $0,83 \pm 0,08$ \\
S13 (reconstrução de fios) & $1,66 \pm 0,14$ \\
S14 (controle de caspas) & $2,82 \pm 0,25$ \\
S15 (reconstrução de fios, sem sulfato) & $2,00 \pm 0,02$ \\
S16 (controle de oleosidade, sem sulfato) & $1,42 \pm 0,14$ \\
\hline
\end{tabular}


Com base nos resultados, foi possível observar que as concentrações de $\mathrm{S}$ para os shampoos avaliados variaram de 0,78 a $2,82 \%$ $\mathrm{m} / \mathrm{v}$, portanto, todas as amostras apresentaram uma concentração de $\mathrm{S}$ menor que 5\%, conforme recomendado pela FDA. O shampoo S5, que tem como principal função o controle da oleosidade, foi o que apresentou a menor concentração de $S(0,78 \% \mathrm{~m} / \mathrm{v})$ o que, provavelmente, deve estar relacionado com a utilização majoritária de surfactantes isentos de $\mathrm{SO}_{4}{ }^{2-}$ em sua composição, bem como de outros compostos isentos de $\mathrm{S}$.

É importante destacar que os shampoos S9 (destinado ao controle de oleosidade) e S14 (destinado ao controle de caspas) foram os que apresentaram as maiores concentrações de S (2,49\% e 2,82\% $\mathrm{m} / \mathrm{v}$, respectivamente). Acredita-se que a concentração obtida para a amostra S9 esteja relacionada a adição de compostos contendo $\mathrm{S}$ com a finalidade de remover a oleosidade, como os surfactantes lauril sulfato de sódio e lauril éter sulfato de sódio que constam no rótulo do produto. A maior concentração de $\mathrm{S}$ foi obtida para o shampoo S14 que tem como finalidade o controle de caspas, e esse resultado pode estar relacionado com a recomendação da FDA, a qual indica uma faixa de concentração de $\mathrm{S}$ neste tipo de produto de 2 a $5 \%$ para a eficiente eliminação de caspas. É importante destacar que os shampoos comercializados como livre de sulfato (S15 e S16) apresentaram concentrações consideráveis de $\mathrm{S}$, as quais foram de $2,00 \%$ e $1,42 \% \mathrm{~m} / \mathrm{v}$, respectivamente, o que possivelmente está relacionado a adição de outros compostos a base de $\mathrm{S}$ aos shampoos. Além disso, shampoos com a mesma ação principal, como o S2 e S6 (realce de cor), apresentaram concentrações de $\mathrm{S}$ que diferiram significativamente, bem como o S5 e 59 para o controle de oleosidade, S8, S11 e S13 para reconstrução de fios e S1 e S10 para cabelos normais. Esses resultados evidenciam a necessidade do desenvolvimento de um método adequado para a determinação da concentração de $\mathrm{S}$ em shampoos, o que foi alcançado através do desenvolvimento desse trabalho, bem como de métodos que viabilizem a determinação das espécies de $\mathrm{S}$ adicionadas nesse tipo de produto.

\section{CONCLUSÃO}

De acordo com os resultados obtidos foi possível verificar que a decomposição por via úmida assistida por radiação micro-ondas em sistema fechado utilizando $\mathrm{HNO}_{3} 7 \mathrm{~mol} \mathrm{~L}^{-1}$ e posterior determinação indireta de $\mathrm{S}$ por espectrofotometria UV-Vis se mostrou um método adequado para o controle de qualidade de shampoos. Além disso, o método proposto não necessitou do uso $\mathrm{HNO}_{3}$ concentrado, reduzindo, assim, os riscos ao analista durante a análise, assim como o consumo de reagentes e a geração de resíduos. Com a utilização desse método foi possível digerir 8 amostras em 40 minutos (20 min de decomposição + 20 min de resfriamento do sistema) e ainda utilizar uma técnica de determinação rápida, precisa e exata, de fácil manuseio e de baixo custo. Também é importante mencionar que o LOD foi adequado a faixa de concentração de $\mathrm{S}$ nas amostras avaliadas, e que apesar das variações observadas na concentração de $S$, todas as amostras estão de acordo com o limite recomendado pela FDA. Nesse sentido, o método proposto pode ser uma alternativa promissora para a aplicação no controle de qualidade de shampoo, tendo em vista a importância de controlar a concentração de S nesse tipo de amostra, as características do método proposto e a carência de métodos que atendam a essa finalidade.

\section{AGRADECIMENTOS}

Os autores agradecem à FAPERGS, a CAPES e ao CNPq pelo apoio financeiro para o desenvolvimento deste trabalho.

\section{REFERÊNCIAS}

1. Gossens, J.; Beleza, um conjunto em harmonia, 1st ed., Harbra: São Paulo, 2004.

2. Gomes, A. L.; O Uso da tecnologia Cosmética no trabalho do profissional Cabeleireiro, 1st ed., Senac: São Paulo, 1999.

3. Bouillon, C.; Clinics in Dermatology 1996, 14, 113.

4. https://www.abihpec.org.br/, acessado em novembro de 2016.

5. Abramovits, W.; Kennedy, A. J.; SKIN: Dermatology for the Clinician 2004, 3, 95.

6. Salvador, A.; Pascual-Martí, M.C.; Aragó, E.; Chisvert, A.; March, J.G.; Talanta 2001, 51, 1171.

7. Miller, H. E.; Arch. Dermatol. Syph. 1935, 31, 516.

8. Leyden, J. J.; McGinley, K. J.; Mills; O. H.; Kyriakopoulos, A. A.; Kligman, A.M.; Cutis 1987, 39, 557.

9. Nitschke, M.; Pastore, G. M.; Quím. Nova 2002, 25, 772.

10. Bamford, J. T. M.; J. Am. Acad. Dermatol. 1983, 8, 211.

11. Andrew, N.; Lin, M. D.; Richard J. Reimer, B. A.; Martin C. M. D.; J. Am. Acad. Dermatol. 1988, 18, 553.

12. http://www.fda.gov/, acessado em março de 2017.

13. Nunes, T. S.; Muller, C. C.; Balestrin, P.; Muller, A. L. H.; Mesko, M. F.; Mello, P. A.; Muller, E. I.; Anal. Methods. 2015, 7, 2129.

14. Lobinski, R.; Marczenko, Z.; Crit. Rev. Anal. Chem. 1992, 23, 55.

15. Galo, A. L.; Colombo, M. F. M.; Quim. Nova 2009, 32, 488

16. Tabatai, M. A.; Environ. Lett. 1974, 7, 237.

17. Krug, F. J.; Rocha, F. R. P.; Métodos de preparo de amostras para análise elementar, 3rd ed., EditSBQ: São Paulo, 2016.

18. Demanze, C.; Rugroff, L.; Karleskind, A.; Parfums, cosmet., aromes 1984, 58, 69.

19. Pathare, M. N.; Sawant A. D.; Anal. Lett. 1995, 28, 317

20. Burguera, M.; Burguera, J. L.; Quim. Anal. 1996, 15, 112.

21. Smith, F. E.; Arsenault E. A.; Talanta 1996, 43, 1207.

22. Hoenig, M.; Talanta 2001, 54, 1021.

23. Azcue, J.; Mudroch, A.; Environ. Anal. Chem. 1994, 12, 211.

24. Melo, L. C. A.; Silva, C. A.; Quím. Nova 2008, 31, 556.

25. Mesko, M. F.; Picoloto, R. S.; Ferreira, L. R.; Costa, V. C.; Pereira, C. M. P.; Colepicolo, P.; Muller, E. I.; Flores, E. M. M.; J. Anal. At. Spectrom. 2014, 30, 260.

26. Flores, E. M. M; Microwave-Assisted Sample Preparation for Trace Element Determination, 1st ed., Elsevier: Amsterdam, 2014.

27. Itoh, H.; Shinbori, Y.; Bull. Chem. Soc. Jpn. 1987, 60, 1327.

28. López-Ruiz, B.; Journal of Cromatography A 2000, 881, 607.

29. Corazza, G.; Henn, A. S.; Mesko, M. F.; Duarte, F. A.; Flores, E. M. M.; Mello, P. A.; J. Braz. Chem. Soc. 2016, 27, 1569.

30. Vogel, A. R.; Análise química quantitativa, 6th ed., LTC - Livros Técnicos e Científicos Ed. S.A.: Rio de Janeiro, 2002.

31. http://www.inmetro.gov.br/, acessado em dezembro de 2016. 